\title{
Itchy E3 ubiquitin-protein ligase promotes neuroblastoma progression in vitro and in vivo
}

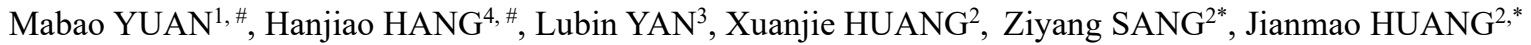 \\ ${ }^{I}$ Department of Pediatric Surgery, Shenzhen Baoan Women's and Children's Hospital, Shenzhen, Guangdong, China, 518133 \\ ${ }^{2}$ Emergency and Disaster Medical Center, the Seventh Affiliated Hospital of Sun Yat-sen University, Shenzhen, Guangdong, \\ China, $518107{ }^{3}$ Department of Pediatric Surgery, the Sixth Affiliated Hospital of Sun Yat-sen University, Guangzhou, \\ Guangdong, China, $510000{ }^{4}$ Shenzhen Longhua Maternity and Child Healthcare Hospital, Shenzhen, Guangdong, China, \\ $518133{ }^{\#}$ Mabao YUAN and Hanjiao HANG contributed to this study equally. \\ *Corresponding authors:Ziyang SANG \& jianmao HUANG.Emergency and Disaster Medical Center, The Seventh Affiliated \\ Hospital of Sun Yat-sen University, No.628, Zhenyuan Rd, Guangming (New) District, Shenzhen, Guangdong, China, \\ 518107. Ziyang Sang, https://orcid.org/0000-0003-3519-7344.
}

Article History Received 3 September 2021; Accepted 25 October 2021; Published 31 December 2021 Cite this Article Mabao YUAN, Hanjiao HANG, Lubin YAN, Xuanjie HUANG, Ziyang SANG2, Jianmao HUANG. Itchy E3 ubiquitin-protein ligase promotes neuroblastoma progression in vitro and in vivo [J].Medical Research, 2021.3(4):12-24, http://doi.org/10.6913/mr.0304.03

Copyright $@ 2021$ Creative Publishing Co., Limited.All rights reserved.Email:mrhk@mrhk.cc

\section{ABSTRACT}

[Objective] Neuroblastoma is the most common pediatric neuroendocrine tumor. Patients with highrisk neuroblastoma have poor clinical outcomes. Understanding the mechanisms underlying neuroblastoma progression could help identify potential therapeutic targets. This study aimed to explore the roles of itchy E3 ubiquitin-protein ligase (ITCH) in neuroblastoma progression using neuroblastoma cell lines and xenograft models of neuroblastoma.

[Methods] ITCH-silencing or overexpressing neuroblastoma cells were established using two different human neuroblastoma cell lines, SK-N-AS and SH-SY5Y. In vitro and in vivo experiments were carried out to determine the effects of ITCH on neuroblastoma cell behaviors. The dualluciferase reporter assay and co-transfection experiments were applied to determine the interaction of ITCH and miR-145-5p during neuroblastoma progression.

[Results] In both cell lines, ITCH overexpression significantly promotes the proliferation, migration, and invasion capacities of neuroblastoma cells, while ITCH silencing with ShITCH suppressed neuroblastoma cell proliferation and induced apoptosis. Moreover, overexpression of ITCH decreased $51 \%$ and 54\% the protein expressions of large tumor suppressor kinase 1 (LATS1), and inhibited 59\% and $66 \%$ the phosphorylation of Yes-associated protein (YAP), concomitant with 2.02-fold and 2.56fold increased expressions of cell proliferation marker Ki67 and 2.51-fold and 2.26-fold elevated levels of anti-apoptosis marker Bcl2 in SK-N-AS and SH-SY5Y cells, respectively. The dualluciferase reporter assay demonstrated that ITCH interacted with miR-145-5p. Further in vitro and xenograft experiments showed that ITCH negatively affected the tumor-suppressive effect of miR$145-5 \mathrm{p}$.

[Conclusion] ITCH promotes neuroblastoma cell proliferation and metastasis by inhibiting LATS1 and promoting YAP nuclear translocation.

KEYWORDS Neuroblastoma; MiR-145-5p; ITCH; Hippo pathway; Yes-associated protein 


\section{Introduction}

Neuroblastoma is the most common neuroendocrine tumor in infants and young children, accounting for $6-10 \%$ of all cancers in children ${ }^{[1-5]}$, and its incidence rate per million children is still increasing [6]. Neuroblastoma occurs outside the skull and often occurs in the nerve tissues of the adrenal glands, chest, abdomen, and pelvis ${ }^{[7,8]}$. Over the past 30 years, the 5 -year survival rates of patients with neuroblastoma have risen from $52 \%$ to $74 \%$, especially in the low-risk group (with up to $92 \% 5$-year survival rate). However, the cure rates in the high-risk group remain marginally improved; around 50$60 \%$ of high-risk patients experience relapse and have poor outcomes ${ }^{[9-11]}$. Therefore, investigating the mechanisms underlying neuroblastoma progression and seeking therapeutic strategies are critical to improving the prognosis of patients.

The itchy E3 ubiquitin-protein ligase (ITCH) is a monomeric protein encoded by the Itch gene. It belongs to homologous to E6-AP carboxy terminus (HECT)-type family and plays a critical role in regulating immunity and cancer ${ }^{[12-15]}$. Due to its unique catalytic activity and substrate recognition properties, ITCH can regulate many transcriptional regulators that are involved in cell growth, differentiation, and apoptosis ${ }^{[13]}$. Currently, investigations on the roles of ITCH on cancer progression are ongoing; however, the role of ITCH on neuroblastoma progression is still unclear. The pre-clinical studies report that, in lung cancer cells, inhibition of ITCH via ITCH silencing RNA decreases proliferation and induces apoptosis ${ }^{[16]}$. In a xenograft model of pancreatic cancer, anti-ITCH siRNA could sensitize pancreatic cancer to gemcitabine in an efficient and specific manner and suppress pancreatic tumor growth ${ }^{[17]}$. Whether ITCH inhibition has similar anti-tumor effects in neuroblastoma remains to be determined.

MicroRNA (miRNA) is a type of single-stranded and highly conserved non-protein encoded small molecule RNA that can regulate the expression of genes. Recent studies have found that there are a wide variety of miRNAs in animals and plants, and their functions cover almost all phenomena in the biological field ${ }^{[18]}$. The main function of miRNA is to target messenger RNA (mRNA) and induce its degradation, thereby regulating gene expression level at the post-transcriptional level ${ }^{[19-21]}$. This process is the key to regulating cell growth, differentiation, apoptosis, and proliferation, and is an important way to regulate tumorigenesis and development. Prior studies demonstrate that the level of ITCH is also regulated by miRNA ${ }^{[5,22]}$. In hepatocellular carcinoma, ITCH is targeted by miR-411 and regulates proliferation ${ }^{[22]}$. It is found that miR-145-5p plays a tumor suppressor role in neuroblastoma ${ }^{[23]}$. However, the regulatory mechanism of miR-145-5p and ITCH in neuroblastoma is lacking to date.

In this study, we aimed to determine the roles of ITCH in neuroblastoma progression using neuroblastoma cell lines and xenograft models of neuroblastoma. We hypothesized that ITCH could exert tumor progression effects in neuroblastoma.

\section{Materials and Methods}

\section{Cell culture}

Two different human neuroblastoma cell lines, SK-N-AS and SH-SY5Y were used in this study ${ }^{[24]}$. The cells were cultured in RPMI-1640 medium (Gibco, Carlsbad, CA, USA) with 10\% FBS and 1\% glutamine. The culture environment was $37{ }^{\circ} \mathrm{C}, 5 \% \mathrm{CO}_{2}$, and $100 \%$ relative humidity.

\section{Cell transfection}

The lentivirus packaging system was used for cell transfection experiments. The ITCH and negative control (NC) were cloned into pCDNA3.1, respectively, and then lentivirus was packaged. MiR-145- 
$5 \mathrm{p}$ mimic, miR-145-5p NC, and shRNA that target ITCH and its NC were purchased from Thermo Fisher Scientific (Waltham, USA). Transfection was performed by Lipofectamine ${ }^{\mathrm{TM}} 2000$ (Invitrogen, Waltham, USA). In brief, $2 \mu \mathrm{L}$ Lipofectamine ${ }^{\mathrm{TM}} 2000$ and plasmid were respectively mixed in $50 \mu \mathrm{L}$ serum-free medium at room temperature for $15 \mathrm{~min}$. The lipid compounds were diluted in $300 \mu \mathrm{L}$ serum-free medium and incubated with the cells at $37{ }^{\circ} \mathrm{C}$ with $5 \% \mathrm{CO}_{2}$ for subsequent experiments.

\section{Real-time polymerase chain reaction (PCR)}

The total RNA was extracted using TRIzol (Sigma, St. Louis, MO, USA) and the purity and concentration of the RNA were detected by Nanodrop (Thermo Fisher Scientific, Waltham, USA). For mRNA detection, reverse transcription was performed using cDNA synthesis kits (Thermo Fisher Scientific, Waltham, USA). SYBR Green PCR Master Mix (Roche, Basle, Switzerland) was used to conduct quantitative PCR experiments. The PCR cycle was as follows: pretreatment at $95^{\circ} \mathrm{C}$ for 10 min; followed by 40 cycles of $94^{\circ} \mathrm{C}$ for $15 \mathrm{~s}, 60^{\circ} \mathrm{C}$ for $1 \mathrm{~min}$, finally at $60^{\circ} \mathrm{C}$ for $1 \mathrm{~min}$ and at $4^{\circ} \mathrm{C}$ for preservation. For miR-145-5p, All-in-One ${ }^{\mathrm{TM}}$ miRNA qRT-PCR Detection Kit 20T/50T (GeneCopoeia, Xi'an, China) was applied to detect the gene expression level. The expression of GAPDH and U6 genes was used for the normalization of mRNA and miRNA, respectively. PCR Detection System (ABI 7500, Life Technology, USA) and comparative cycle threshold $\left({ }^{\Delta \Lambda} \mathrm{Ct}\right)$ were applied to calculate the fold change.

\section{Western blot}

The proteins were extracted by protein lysate and the concentration and purity were detected via the BCA kit. Then the proteins were separated using SDS-PAGE (110 V, $100 \mathrm{~min})$. The proteins were transferred to PVDF membranes ( $90 \mathrm{~V}, 90 \mathrm{~min}$ ). The PVDF membrane was blocked in 5\% non-fat milk for $1 \mathrm{~h}$ at room temperature. The anti-ITCH (Abcam, San Francisco, USA, ab108515), antiLAST1 (ab70561), anti-YAP (ab52771), anti-p-YAP (ab76252), anti-Ki67 (ab16667), anti-Bc12 (ab196495), anti-GAPDH (ab9485), $\beta$-actin (ab8226) and Histone H3 (ab176842) diluted at 1:500 were added and incubated overnight at $4^{\circ} \mathrm{C}$. Then the HRP labeled goat-anti-rabbit secondary antibody was diluted at 1:5000 and added at room temperature for $2 \mathrm{~h}$. Protein blots band was detected by Pierce ${ }^{\mathrm{TM}}$ ECL plus western blotting substrate (Thermo Fisher, Waltham, USA) in ChemiDoc MP (Bio-Rad, California, USA).

\section{Cell counting kit 8 (CCK-8) assay}

CCK-8 viability assay was used to measure cell proliferation. First, $2 \times 10^{4}$ cells $(100 \mu \mathrm{L})$ were seeded in 96-well plates. After cultured for $1 \mathrm{~d}, 2 \mathrm{~d}, 3 \mathrm{~d}, 4 \mathrm{~d}$, and $5 \mathrm{~d}$, respectively, $10 \mu \mathrm{L}$ of CCK-8 (Beyotime Institute of Biotechnology, Beijing, China) was added to the cells and cultured at $37{ }^{\circ} \mathrm{C}$ for another $2 \mathrm{~h}$. The plates were then read by a microplate reader (Tecan Infinite M200 Microplate Reader; LabX, Männedorf, Switzerland). The optical density (OD) was measured at $450 \mathrm{~nm}$.

\section{Flow cytometry assay}

Apoptosis rates were tested using flow cytometry (BD FACSCalibur, Becton Dickinson, USA) with Annexin V-FITC/PI kit (Sanjian Biological Technology Co., Ltd., Chongqing, China). The reagents were added according to the manufacturer's instructions. The percentage of Annexin $\mathrm{V}+$ cells (Q2+Q3) was calculated as the apoptotic rate.

\section{Wound healing assay}

The cells were digested and seeded in 12-well plates $\left(1 \times 10^{5}\right.$ cells per well $)$. Then the cells were cultured until about $90 \%$ confluence. Scratching the cells from top to bottom using a $200 \mu \mathrm{L}$ pipette 
tip to mimic a wound. The cells were then cultured for another $24 \mathrm{~h}$ under the same conditions. The images of the cells were obtained using optical microscopy.

\section{Invasion assay}

The Matrigel was added to the upper chamber of the Transwell chamber, and the complete medium was added to the bottom chamber. $5 \times 10^{4}$ cells were added to the upper chamber for $48 \mathrm{~h}$. After $48 \mathrm{~h}$, the cells that did not penetrate the bottom chamber were washed away. The cells were then fixed with $20 \%$ methanol and stained with $0.1 \%$ crystal violet. Five fields were randomly selected under a microscope (Olympus, Japan), and the number of cells invading the bottom chamber of each field was counted and the average value was calculated.

\section{Dual-luciferase reporter assay}

Wild-type (WT) / mutated (MUT) ITCH and miR-145-5p mimic were both cloned into pMIRREPORT Luciferase vectors (Ambion, Thermo Fisher Scientific, Waltham, MA). SK-N-AS and SHSY5Y cells were transfected with both vectors using Lipofectamine 2000 for $24 \mathrm{~h}$. The Dual Luciferase-Reporter 1000 Assay System (Promega, Madison, WI) was used to evaluate luciferase activity.

\section{Xenograft studies}

The animal experiments were approved by the Institutional Animal Care and Use Committee (IACUC) of Sun Yat-sen University. The cells were injected subcutaneously into the right hind flank of 5-week-old male BALB/c nude mice. When the tumor volume reached about $100 \mathrm{~mm}^{3}$, the mice were euthanized and the size of tumors was measured.

\section{Statistical analysis}

All experiments were repeated three times. All statistical analyses were performed using GraphPad Prism 7 (GraphPad Software, Inc., USA). Statistical significance between the two groups was performed using the Student's $t$-test. Statistical significance among three or more groups was determined by a one-way analysis of variance (ANOVA), followed by a post hoc Tukey test. All experimental data are presented as the mean \pm standard deviation (SD). The P-value less than 0.05 was considered statistically significant.

\section{Results}

\section{ITCH promotes proliferation and metastasis of neuroblastoma cells}

To determine the role of ITCH in neuroblastoma cell behaviors, we examined cell proliferation, survival, migration, and evasion in two different neuroblastoma cell lines (SK-N-AS and SH-SY5Y) with ITCH overexpression or silencing. First, we verified the efficiency of ITCH overexpression and silencing (Figure 1A-D). The western blot test results confirmed that the levels of ITCH protein were increased by 2.03 -fold and 2.18-fold after overexpression via lentivirus packaging in SK-N-AS and SH-SY5Y cells, respectively (Figure 1A-B). Three shRNAs were designed to inhibit ITCH; among them, the shITCH-1 reduced ITHC protein expression about 0.24 and 0.23 times the baseline level in SK-N-AS and SH-SY5Y cells, respectively (Figure 1C-D). Thus, it was selected and used for the following experiments. Next, the effects of ITCH on neuroblastoma cell biological behaviors were evaluated. We observed that ITCH overexpression was linked to enhanced proliferation and reduced apoptosis of neuroblastoma cells while silencing of ITCH significantly inhibited their proliferation and promoted apoptosis (Figure 1E-H). Further, the wound healing assay and invasion assay demonstrated that neuroblastoma cells with ITCH overexpression showed increased migration (SK-N- 
AS: $26.67 \pm 4.17 \%$ vs. $80.01 \pm 10.21 \%$; SH-SY5Y: $24.17 \pm 5.02 \%$ vs. $73.33 \pm 5.83 \%$, both $\mathrm{p}<0.05$ ) and invasion capabilities (SK-N-AS: 1.84-fold vs. 0.55-fold; SH-SY5Y: 1.92-fold vs.0.56-fold, both $\mathrm{p}<0.05$ ), and these capabilities were suppressed in ITCH silenced cells by shITCH (Figure 1I-L). These data suggested that ITCH had tumor-promoting effects in neuroblastoma.

A

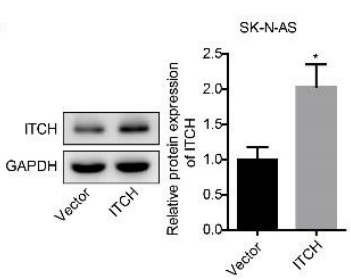

E SK-NAS
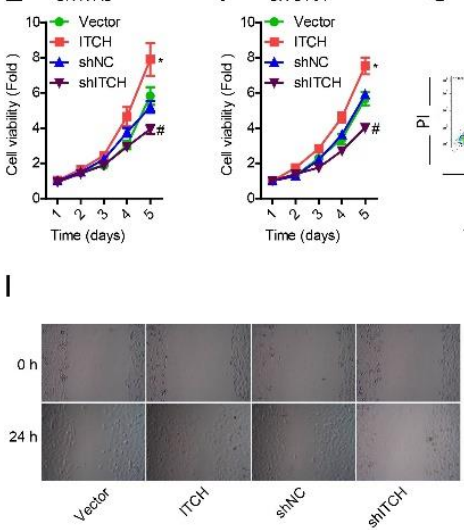

$\mathrm{K}$
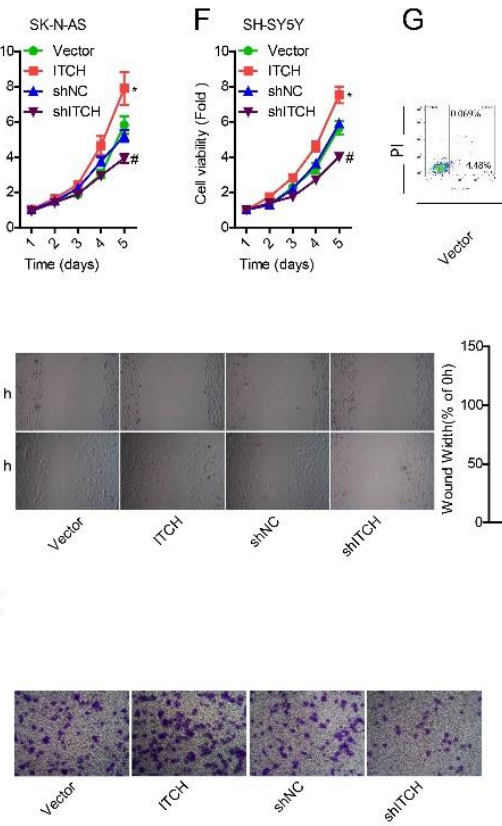

B

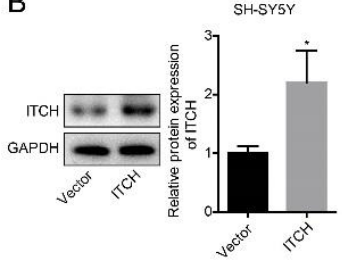

C

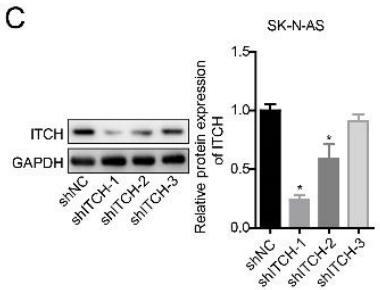

D

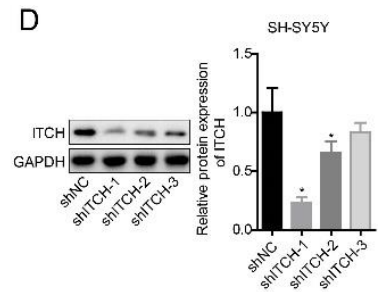

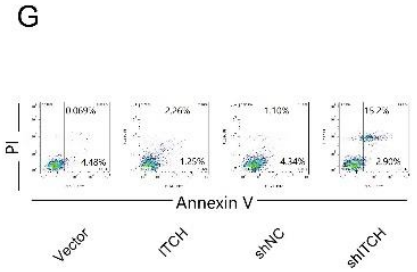

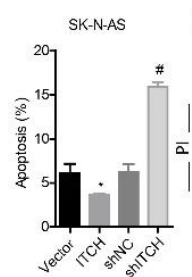

$\mathrm{H}$
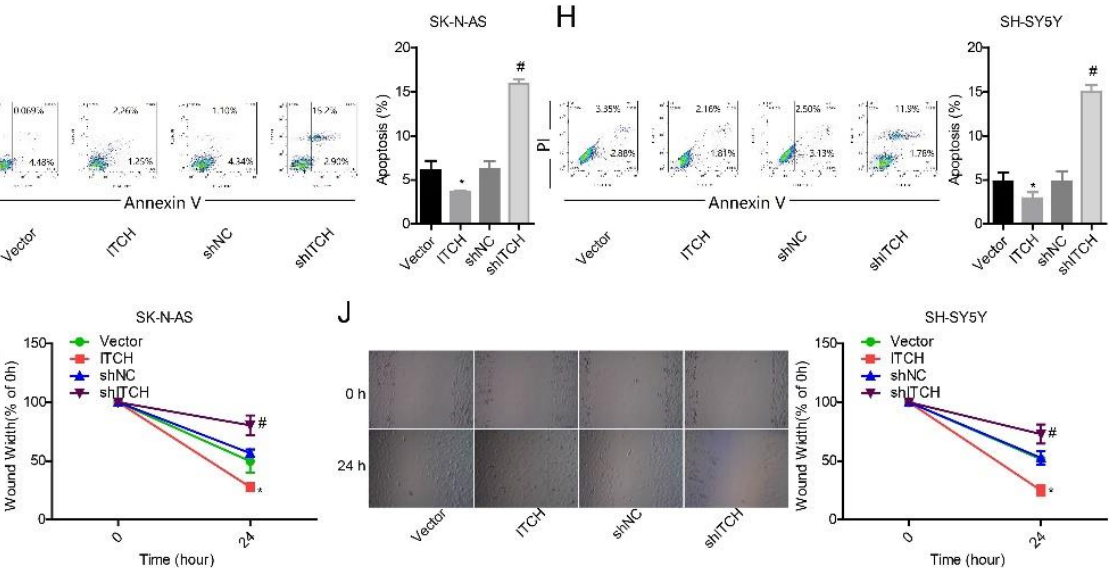

$J$
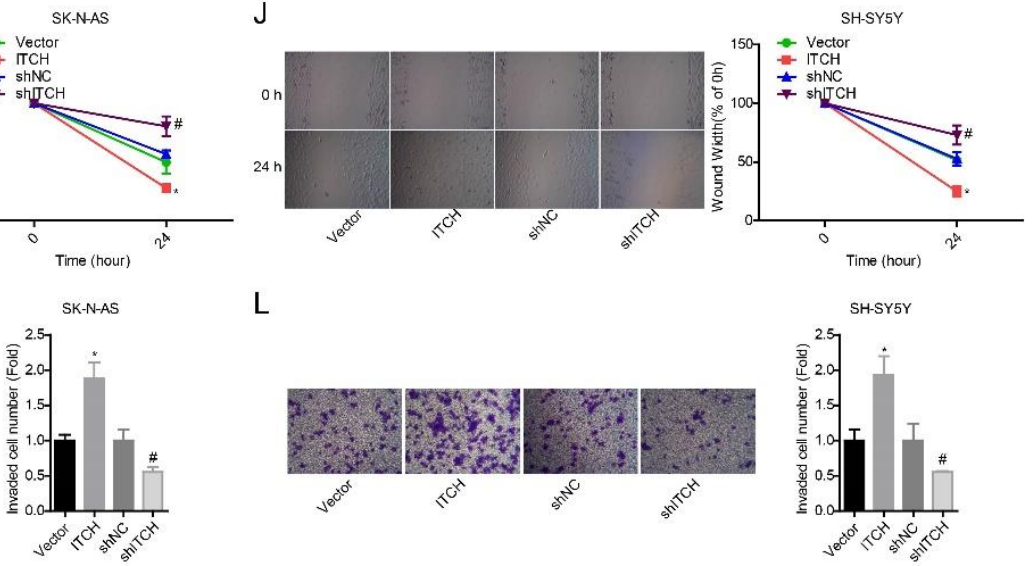

$\mathrm{L}$

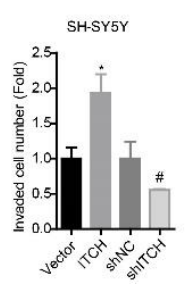

Figure 1. Overexpression of ITCH promotes the proliferation and metastasis of neuroblastoma cells.

(A-B) The effects of ITCH overexpression plasmid on the level of ITCH protein in two different neuroblastoma cell lines (SK-N-AS and SH-SY5Y). (C-D) The levels of ITCH protein in the SK-N-AS and SH-SY5Y cells after silencing ITCH with shITCH-1, shITCH-2, or shITCH-3. (E-F) The effects of ITCH overexpression or silencing on cell proliferation. (G-H) The effects of ITCH overexpression or silencing on cell apoptosis. (I-J) The effects of ITCH overexpression or silencing on cell migration. (K-L) The effects of ITCH overexpression or silencing on cell invasion. ${ }^{*} P<0.05$ vs. vector group. ${ }^{\sharp} P<0.05$ vs. shNC group.

\section{ITCH regulates the Hippo pathway and promotes YAP nuclear translocation}

To investigate the mechanism by which ITCH promotes neuroblastoma, we examined the protein levels of key components of the Hippo signaling, an emerging pathway involved in drug resistance of neuroblastoma ${ }^{[25]}$. Prior studies have shown that the Hippo-Yes-associated protein (YAP) pathway is involved in regulating neuroblastoma progression ${ }^{[26,27]}$ and is regulated by ITCH ${ }^{[28,29]}$. In the Hippo pathway, signal transduction is through activating serine/threonine kinase 4 (STK4) and large tumor suppressor kinase 1 (LATS1) proteins and eventually leads to the phosphorylation of YAP. After being phosphorylated, YAP strands in the cytoplasm and is degraded. ITCH can mediate the ubiquitination 
degradation of LATS1 ${ }^{[29]}$, thereby inhibiting YAP phosphorylation and promoting YAP nuclear translocation, and promoting breast cancer metastasis ${ }^{[29,30]}$.

We observed that in ITCH overexpressed SK-N-AS and SH-SY5Y cells, the expression levels of large tumor suppressor kinase (LATS-1) were decreased 51\% and 54\%, respectively (Figure 2A). 59\% and $66 \%$ reductions were also found in the level of phosphorylated YAP in ITCH overexpressed SKN-AS and SH-SY5Y cells, respectively (Figure 2B). Further experiments indicated a partial translocation of YAP protein into the nucleus (Figure 2C-D). Moreover, we observed 2.02-fold and 2.56-fold increases in protein expressions of the proliferation marker Ki67 in ITCH overexpressing SK-N-AS and SH-SY5Y cells, respectively (Figure 2A). 2.51-fold and 2.26-fold elevations were presented in anti-apoptosis marker Bcl2 in ITCH overexpressing SK-N-AS and SH-SY5Y cells, respectively (Figure 2B). These results suggested that ITCH may inhibit the phosphorylation of YAP by regulating the Hippo pathway, promote YAP entry into nuclear to exert transcriptional regulation, and promote the progression of neuroblastoma.

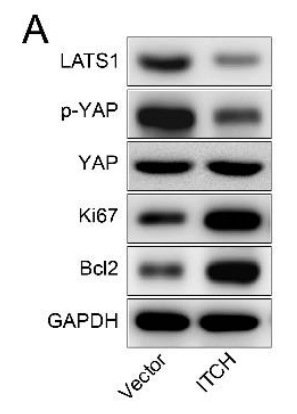

C

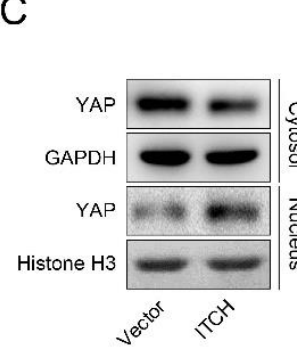

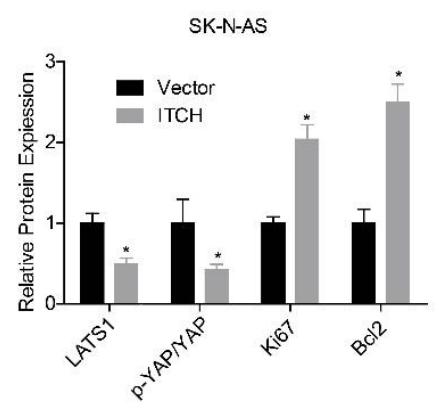

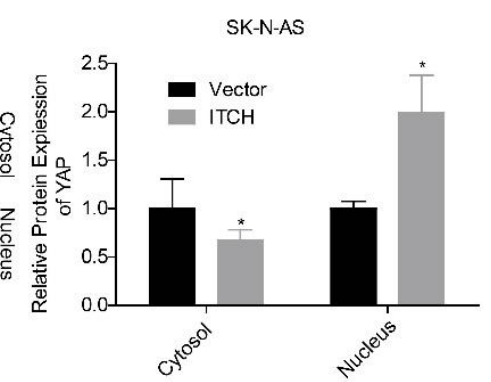

B

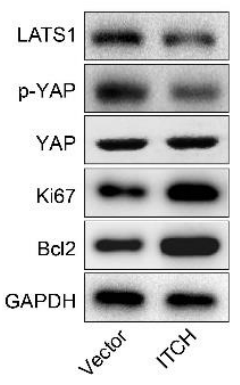

D

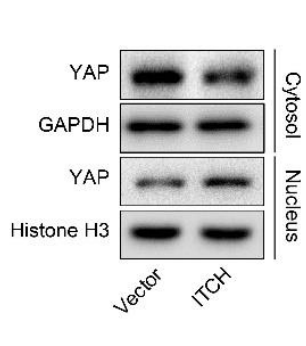

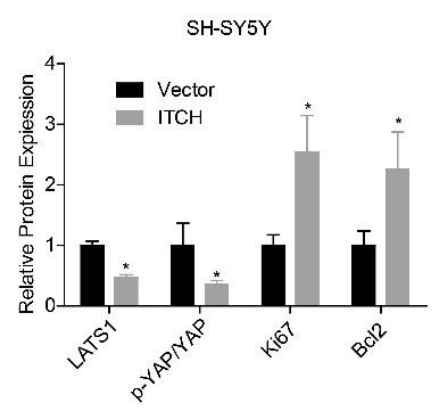

SH-SY5Y

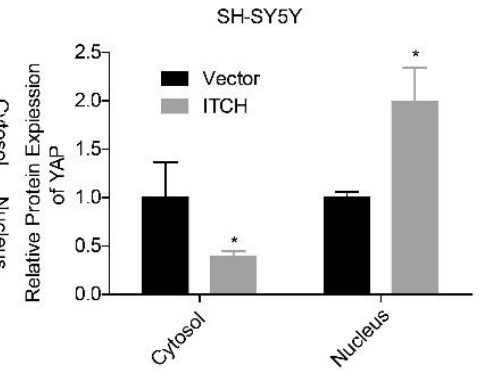

Figure 2. ITCH inhibits the Hippo pathway and YAP phosphorylation and promotes YAP nuclear translocation. (A-B) The effects of ITCH overexpression on the protein levels of LATS1, YAP, Ki67, and Bcl2 in the SK-N-AS and SH-SY5Y cell lines. (C-D) The effects of ITCH overexpression on YAP nuclear translocation in the SK-N-AS and SH-SY5Y cells. ${ }^{*} P<0.05$ vs. vector group.

\section{ITCH binds to miR-145-5p}

It has been reported that ITCH has miRNA binding sites ${ }^{[31]}$. Several miRNAs can be bound to the $3^{\prime}-$ untranslated regions (UTR) of ITCH and regulate the ITCH expression in tumors ${ }^{[32]}$. The miR-145 is a critical tumor suppressor in neuroblastoma ${ }^{[23,33]}$. We, therefore, sought to determine whether miR145 could bind to ITCH in neuroblastoma cells. The binding site of miR-145-5p and ITCH was shown in Figure 3A. The dual-luciferase report experimental results demonstrated that miR-145-5p could directly bind to 3'UTR of ITCH mRNA in SK-N-AS and SH-SY5Y cells (Figure 3B-E). Moreover, overexpression of miR-145-5p significantly inhibited ITCH mRNA expressions (47.06 $\pm 5.88 \%$ in SK$\mathrm{N}$-AS cells and $40.34 \pm 5.04 \%$ in SH-SY5Y cells) (Figure 3F, G) and protein levels $(49.15 \pm 16.95 \%$ in SK-N-AS cells and $43.22 \pm 11.02 \%$ in SH-SY5Y cells) (Figure 3H, I). 


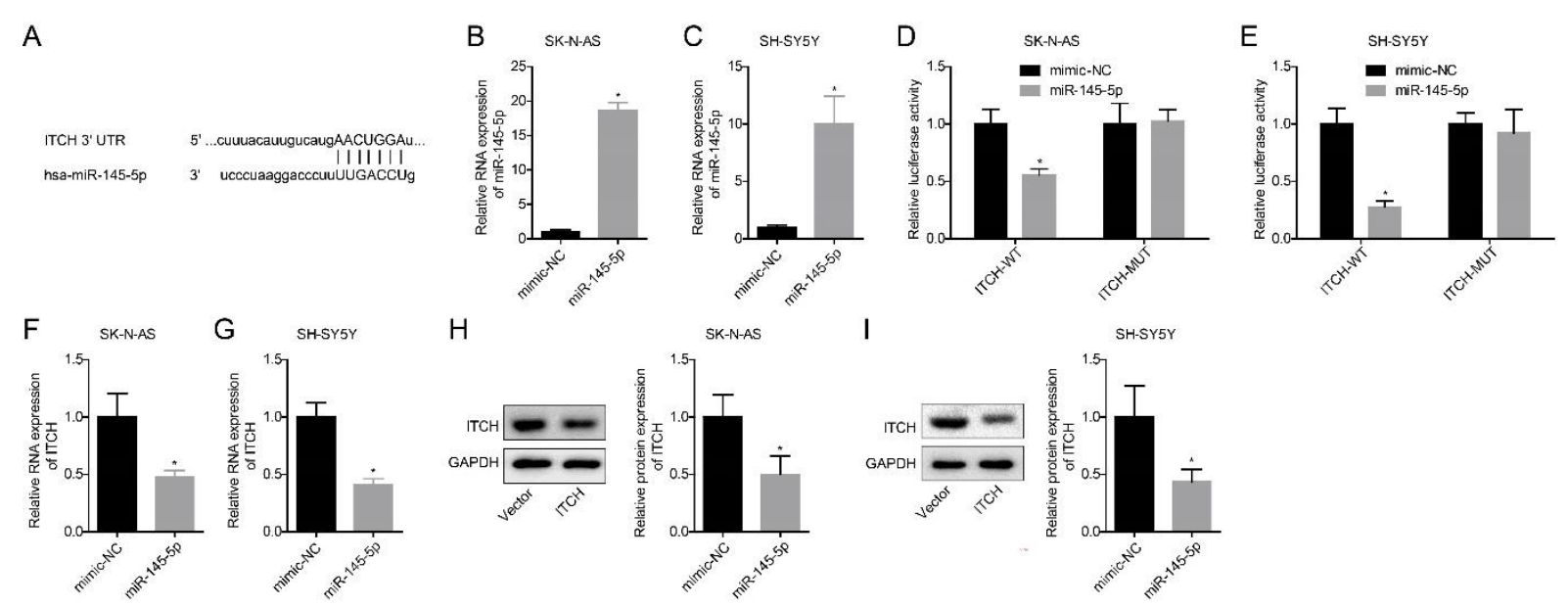

Figure 3. The expression of ITCH is regulated by miR-145-5p. (A) Prediction results of miR-145-5p and ITCH. (B-C) Transfection efficiency of miR-145-5p mimic using the SK-N-AS and SH-SY5Y cell lines. (D-E) Dual-fluorescein report experiment showed that miR-145-5p and ITCH could be targeted binding in SK-N-AS and SH-SY5Y cells. (F-I) The effects of miR-145-5p mimic the ITCH expression in the SK-N-AS and SH-SY5Y cell lines. ${ }^{*} P<0.05$ vs. mimic-NC group.

MiR-145-5p inhibits neuroblastoma progression by regulating ITCH expression in vitro and in vivo

To determine whether miR-145-5p signals through ITCH in neuroblastoma suppression, SK-N-AS and SH-SY5Y cells that were transfected with miR-145-5p alone, miR-145-5p+ITCH, and ITCH alone were used. The western blot results confirmed that miR-145-5p could target the expression levels of ITCH protein (Figure 4A-B). Moreover, overexpression of miR-145-5p significantly inhibited the neuroblastoma cell proliferation (Figure 4C, D) and promoted their apoptosis (Figure 4E, F), along with decreased migration (Figure $4 \mathbf{G}, \mathbf{H}$ ) and invasion (Figure 4I, J), while overexpression of ITCH partially reversed the effects by miR-145-5p (Figure 4C-4J). These data verified that the cancer-promoting effects of ITCH were regulated by miR-145-5p.

To further verify the in vitro results, we took advantage of tumor xenograft models, using miR$145-5 p$ alone or miR-145-5p+ITCH overexpressing neuroblastoma cells engraftment into nude mice. As a result, we found that miR-145-5p significantly inhibited tumor growth, and ITCH overexpression interrupted the miR-145-5p's suppressive impact on tumor growth (Figure 5A-B). In tumor tissues, the ITCH protein level was significantly inhibited to $53.01 \pm 3.00 \%$ and $48.48 \pm 6.06 \%$, and in contrast, the LATS1 protein level increased to 1.81-fold and 2.50-fold in SK-N-AS and SH-SY5Y cells of miR145-5p group, respectively (Figure 5E-F). Also, the ITCH protein level in the miR-145-5p+ITCH group was significantly $61 \%$ and $45 \%$ higher than that of the SK-N-AS and SH-SY5Y cells of miR145-5p group, while their LATS1 levels were 90\% and 147\% lower, respectively (Figure 5E-F). Taken together, these data confirm that miR-145-5p signals through ITCH and Hippo pathway to suppress neuroblastoma and overexpression of ITCH may negatively affect the tumor-suppressive effect of miR-145-5p. 
A

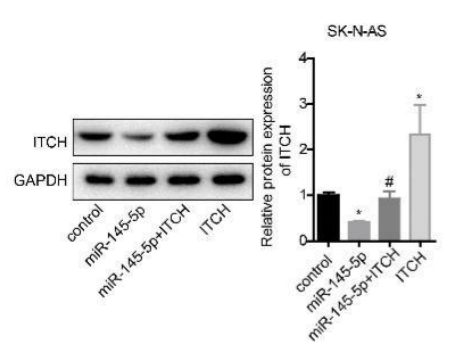

E

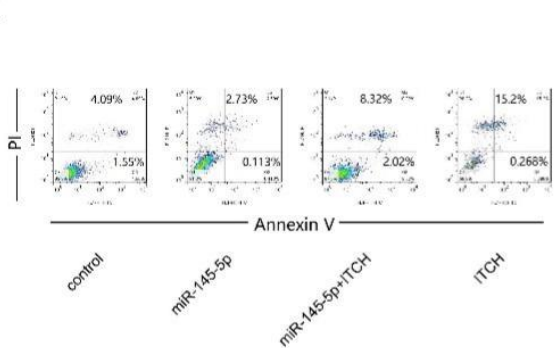

G

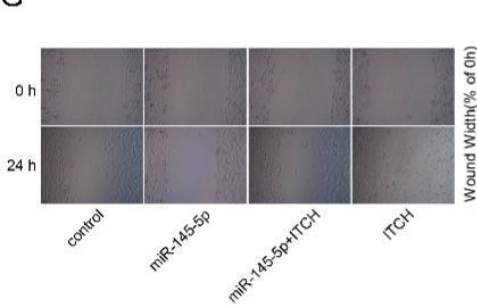

I

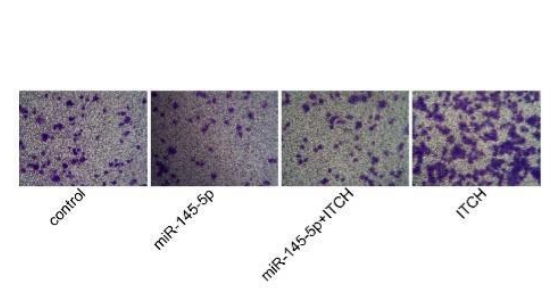

B

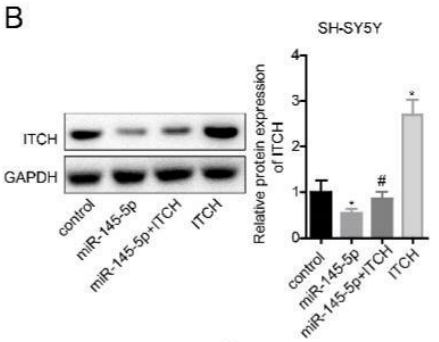

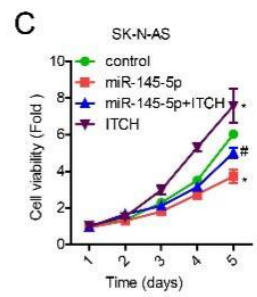

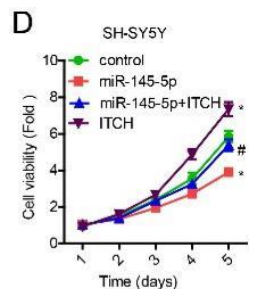

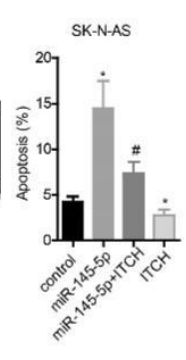

SK-N-AS

F
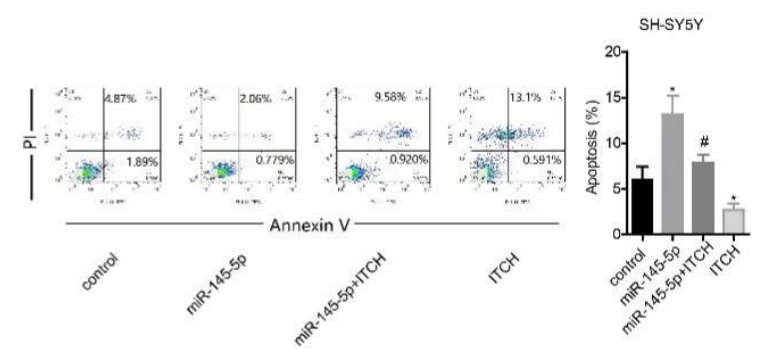

$\mathrm{H}$

SH-SYSY

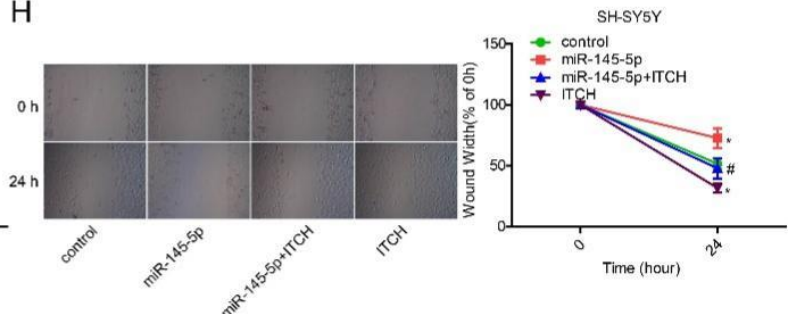

$J$

SH-SYSY
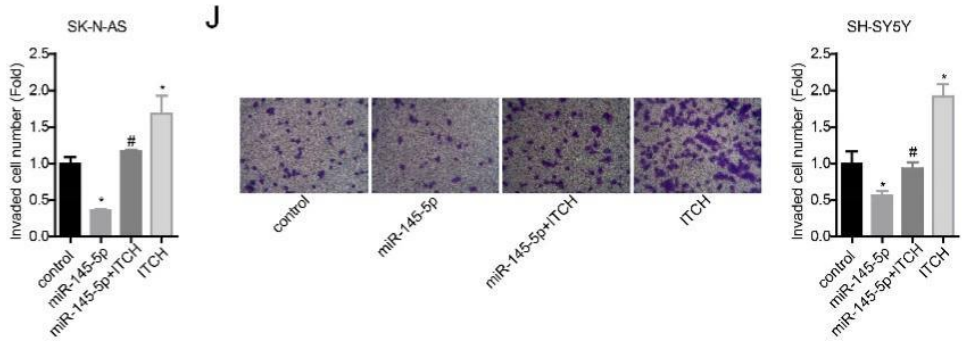

Figure 4. MiR-145-5p inhibits neuroblastoma through targeted inhibition of ITCH expression in vitro. (A-

B) The expression levels of ITCH protein in each group after transfection. (C-D) Effects of overexpression of miR-145-5p and/or ITCH on the proliferation of SK-N-AS and SH-SY5Y cells. (E-F) Effects of overexpression of miR-145-5p and/or ITCH on cell apoptosis. (G-H) Effects of overexpression of miR-145-5p and/or ITCH on cell migration. (I-J) Effects of overexpression of miR-145-5p and/or ITCH on cell invasion. ${ }^{*} P<0.05$ vs. control group. ${ }^{\sharp} P<0.05$ vs. miR-145-5p group. 

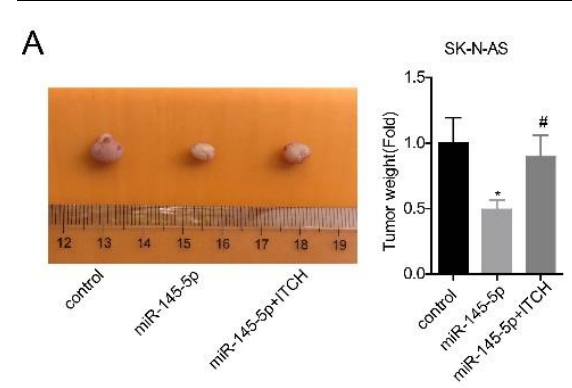

B
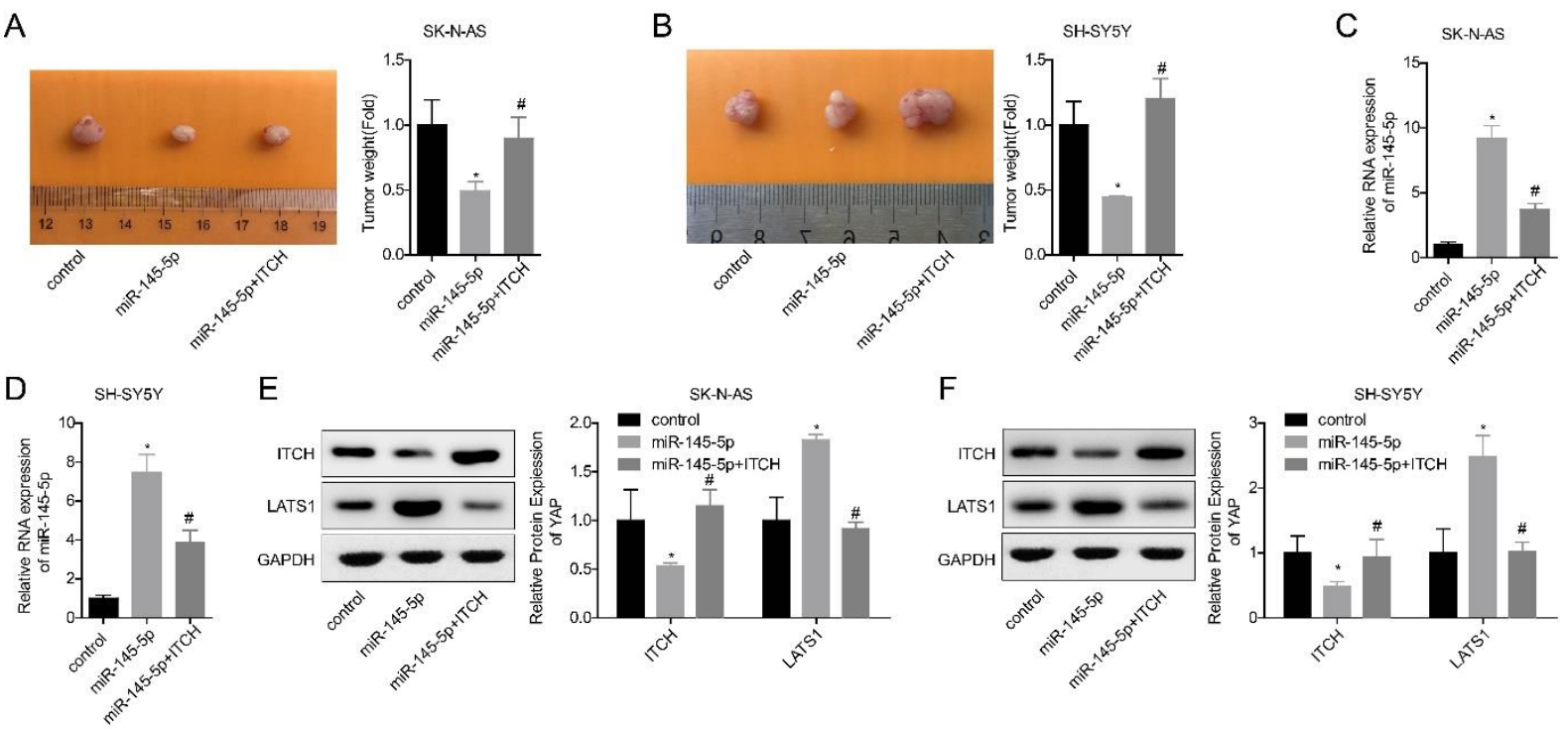

Figure 5. MiR-145-5p inhibits neuroblastoma through targeted inhibition of ITCH in vivo. (A-B) The effects of miR-145-5p via ITCH on tumor growth via xenograft experiment. (C-D) The levels of miR-145-5p in tumor tissues of each group. (E-F) The protein levels of LATS1 and ITCH in each group were compared. ${ }^{*} P<$ 0.05 vs. control group. ${ }^{\#} P<0.05$ vs. miR-145-5p group.

\section{Discussion}

ITCH has a regulatory effect on a variety of proteins, thereby regulating different signaling pathways, promoting or inhibiting tumorigenesis and progression ${ }^{[34]}$. ITCH has a dual effect on tumors. On one hand, ITCH induces disheveled phosphorylation and degradation to inhibit the Wnt pathway and inhibit the progression of melanoma ${ }^{[35]}$. On the other hand, It is confirmed that ITCH can inhibit Hippo pathway by binding to LATS1 and triggering ubiquitination degradation, thereby inducing epithelial-mesenchymal transition to cause tumorigenesis ${ }^{[28]}$. The role of ITCH in neuroblastoma is still uncertain. In this study, ITCH overexpression and silenced neuroblastoma cell models were established, and it was found that the up-regulation of ITCH protein level promoted the proliferation and invasion of neuroblastoma cells, and inhibited apoptosis. Correspondingly, down-regulating the level of ITCH protein had a tumor-suppressing effect. This suggested that in neuroblastoma, ITCH had a cancer-promoting effect.

To further analyze the mechanism by which ITCH promotes neuroblastoma, we analyzed pathways with tumor-suppressing effects and found that ITCH could cause a decrease in LATS1 protein levels. LATS1 is a central regulator in the Hippo pathway ${ }^{[36]}$. When LATS1 protein is activated by upstream mammalian Ste20-like 1/2 (MST1/2) and scaffold protein Salvador 1 (SAV1), it can promote the phosphorylation of YAP ${ }^{[37]}$. After being phosphorylated, YAP cannot enter the nucleus to perform transcriptional regulation, leading to antitumor effects ${ }^{[38]}$. The results of this study also showed that after overexpression of ITCH, the levels of YAP protein entering the nucleus increased, and the levels of proto-oncogene transcription levels and protein expression levels also increased. The latest research shows that YAP has a cancer-promoting effect in neuroblastoma ${ }^{[39]}$. In vitro study also shows that YAP can regulate mitochondrial fission, inhibit neuroblastoma cell apoptosis, and promote survival ${ }^{[40]}$. This suggests that in neuroblastoma, ITCH may reduce YAP phosphorylation and promote nuclear translocation by inhibiting LATS1 and Hippo pathways and play a role in promoting cell proliferation and invasion. This mechanism is also revealed in breast cancer ${ }^{[41,42]}$. 
A large number of studies in recent years have shown that miR-145-5p has tumor-suppressing effects in various types of cancer, including glioblastoma ${ }^{[43]}$, gastric cancer ${ }^{[44]}$, and breast cancer ${ }^{[45]}$. In neuroblastoma, in vitro and in vivo experiments have confirmed that miR-145-5p can inhibit the proliferation, migration and invasion of neuroblastoma cells ${ }^{[23,33]}$. Moreover, miR-145 may also inhibit the aggressiveness of cisplatin-resistant neuroblastoma cells by regulating autophagy ${ }^{[46]}$. The results of this study showed that miR-145-5p directly bound to the 3'UTR of ITCH mRNA, and eventually caused the down-regulation of ITCH protein. In addition, the anti-neuroblastoma effects of miR-145-5p were achieved by inhibiting ITCH and YAP nuclear translocation. These results reveal that miR-145-5p could regulate the ITCH and signal through the Hippo pathway to suppress tumor cell proliferation and that overexpression of ITCH may negatively affect the tumor-suppressive effect of miR-145-5p. The scheme of proposed mechanisms is summarized in Figure 6.

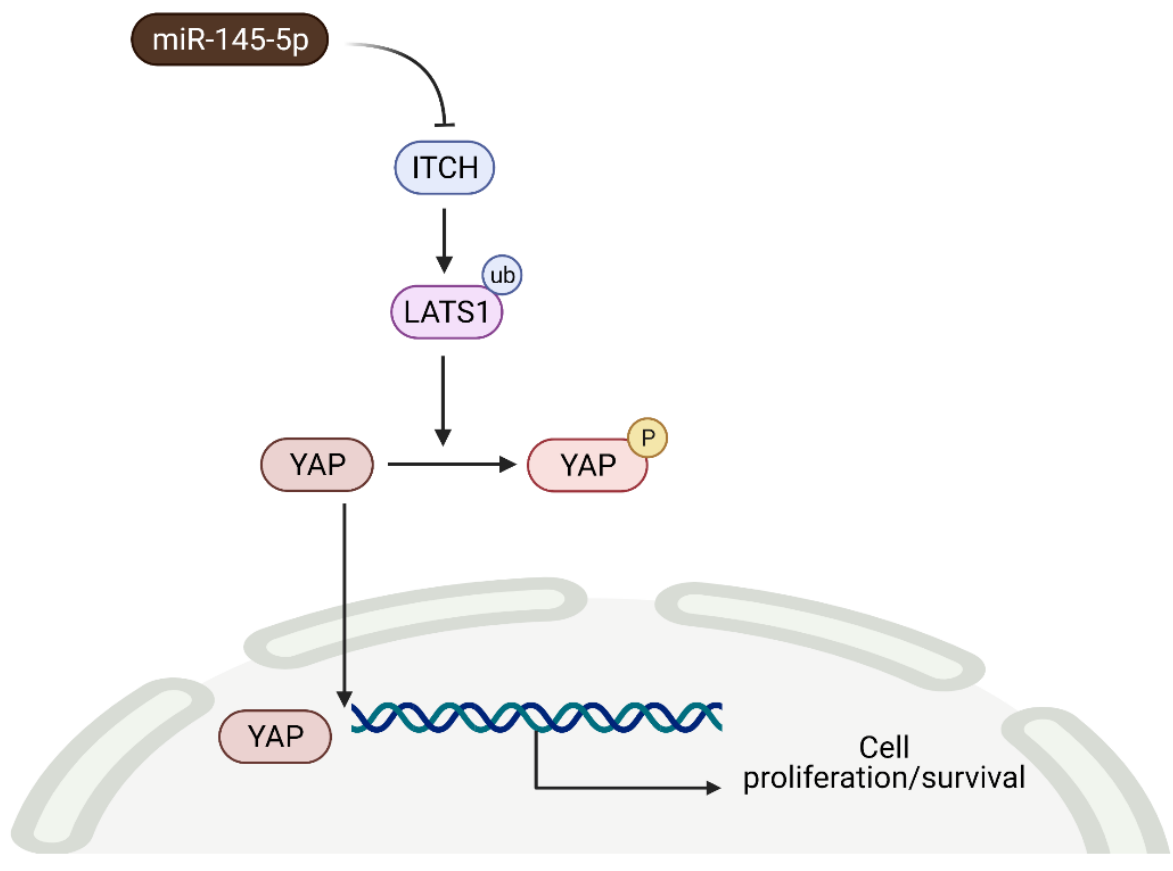

Figure 6. The scheme of proposed mechanism underlying miR-145-5p and ITCH in neuroblastoma. MiR145-5p negatively regulates ITCH expression. ITCH can promote the ubiquitination degradation of LATS1, inhibit the YAP phosphorylation, and enhance YAP translocation into nucleus, thereby mediating cell proliferation. ITCH, itchy E3 ubiquitin-protein ligase; LATS1, large tumor suppressor kinase 1; YAP, yesassociated protein; ub, ubiquitination; P, phosphorylation. The figure was created with BioRender.com.

\section{Conclusion}

In conclusion, ITCH can inhibit YAP phosphorylation by inhibiting the expression of LATS1 and promoting YAP nuclear translocation, thereby promoting the progression of neuroblastoma. Targeting ITCH may help inhibit neuroblastoma progression. More studies using clinical samples are warranted to validate these preclinical results.

\section{Data Availability}

The data used to support the findings of this study are available from the corresponding author upon reasonable request. 


\section{Conflict of interest}

The authors declare no conflict of interest.

\section{References:}

1. E. Ward, C. DeSantis, A. Robbins, B. Kohler and A. Jemal, "Childhood and adolescent cancer statistics, 2014," CA Cancer J Clin, vol. 64, no. 2, pp. 83-103, 2014.

2. N. C. Colon and D. H. Chung, "Neuroblastoma," Adv Pediatr, vol. 58, no. 1, pp. 297-311, 2011.

3. P. P. Bao, K. Li, C. X. Wu, Z. Z. Huang, C. F. Wang, Y. M. Xiang, P. Peng, Y. M. Gong, X. M. Xiao and Y. Zheng, "[Recent incidences and trends of childhood malignant solid tumors in Shanghai, 2002-2010]," Zhonghua Er Ke Za Zhi, vol. 51, no. 4, pp. 288-294, 2013.

4. Y. L. Liu, W. C. Lo, C. J. Chiang, Y. W. Yang, M. Y. Lu, W. M. Hsu, W. L. Ho, M. J. Li, J. S. Miser, D. T. Lin and M. S. Lai, "Incidence of cancer in children aged 0-14 years in Taiwan, 1996-2010," Cancer Epidemiol, vol. 39, no. 1, pp. 21-28, 2015.

5. L. Wan, L. Zhang, K. Fan, Z. X. Cheng, Q. C. Sun and J. J. Wang, "Circular RNA-ITCH Suppresses Lung Cancer Proliferation via Inhibiting the Wnt/beta-Catenin Pathway," Biomed Res Int, vol. 2016, pp. 1579490 , 2016.

6. F. Berthold, C. Spix, P. Kaatsch and F. Lampert, "Incidence, Survival, and Treatment of Localized and Metastatic Neuroblastoma in Germany 1979-2015," Paediatr Drugs, vol. 19, no. 6, pp. 577-593, 2017.

7. W. G. He, Y. Yan, W. Tang, R. Cai and G. Ren, "Clinical and biological features of neuroblastic tumors: A comparison of neuroblastoma and ganglioneuroblastoma," Oncotarget, vol. 8, no. 23, pp. 37730-37739, 2017

8. L. E. Ritenour, M. P. Randall, K. R. Bosse and S. J. Diskin, "Genetic susceptibility to neuroblastoma: current knowledge and future directions," Cell Tissue Res, vol. 372, no. 2, pp. 287-307, 2018.

9. J. M. Maris, "Recent advances in neuroblastoma," N Engl J Med, vol. 362, no. 23, pp. 2202-2211, 2010.

10. W. B. London, V. Castel, T. Monclair, P. F. Ambros, A. D. Pearson, S. L. Cohn, F. Berthold, A. Nakagawara, R. L. Ladenstein, T. Iehara and K. K. Matthay, "Clinical and biologic features predictive of survival after relapse of neuroblastoma: a report from the International Neuroblastoma Risk Group project," J Clin Oncol, vol. 29, no. 24, pp. 3286-3292, 2011.

11. B. A. Tajudeen, A. Arshi, J. D. Suh, M. St John and M. B. Wang, "Importance of tumor grade in esthesioneuroblastoma survival: a population-based analysis," JAMA Otolaryngol Head Neck Surg, vol. 140, no. 12, pp. 1124-1129, 2014.

12. N. J. Lohr, J. P. Molleston, K. A. Strauss, W. Torres-Martinez, E. A. Sherman, R. H. Squires, N. L. Rider, K. R. Chikwava, O. W. Cummings, D. H. Morton and E. G. Puffenberger, "Human ITCH E3 ubiquitin ligase deficiency causes syndromic multisystem autoimmune disease," Am J Hum Genet, vol. 86, no. 3, pp. 447453, 2010.

13. G. Melino, E. Gallagher, R. I. Aqeilan, R. Knight, A. Peschiaroli, M. Rossi, F. Scialpi, M. Malatesta, L. Zocchi, G. Browne, A. Ciechanover and F. Bernassola, "Itch: a HECT-type E3 ligase regulating immunity, skin and cancer," Cell Death Differ, vol. 15, no. 7, pp. 1103-1112, 2008.

14. E. K. Moser and P. M. Oliver, "Regulation of autoimmune disease by the E3 ubiquitin ligase Itch," Cell Immunol, vol. 340, pp. 103916, 2019.

15. B. Yang, J. Zhao, T. Huo, M. Zhang and X. Wu, "Effects of CircRNA-ITCH on proliferation and apoptosis of hepatocellular carcinoma cells through inhibiting Wnt/beta-catenin signaling pathway," J BUON, vol. 25, no. 3, pp. 1368-1374, 2020.

16. P. F. Li and Q. G. Zhang, "Inhibition of ITCH Suppresses Proliferation and Induces Apoptosis of Lung Cancer Cells," Cell Physiol Biochem, vol. 48, no. 4, pp. 1703-1709, 2018.

17. M. de la Fuente, M. C. Jones, M. J. Santander-Ortega, A. Mirenska, P. Marimuthu, I. Uchegbu and A. Schatzlein, "A nano-enabled cancer-specific ITCH RNAi chemotherapy booster for pancreatic cancer," Nanomedicine, vol. 11, no. 2, pp. 369-377, 2015.

18. T. X. Lu and M. E. Rothenberg, "MicroRNA," J Allergy Clin Immunol, vol. 141, no. 4, pp. 1202-1207, 2018. 
19. R. Baffa, M. Fassan, S. Volinia, B. O'Hara, C. G. Liu, J. P. Palazzo, M. Gardiman, M. Rugge, L. G. Gomella, C. M. Croce and A. Rosenberg, "MicroRNA expression profiling of human metastatic cancers identifies cancer gene targets," J Pathol, vol. 219, no. 2, pp. 214-221, 2009.

20. Y. Mu, Q. Wang, L. Tan, L. Lin and B. Zhang, "microRNA-144 inhibits cell proliferation and invasion by directly targeting TIGAR in esophageal carcinoma," Oncol Lett, vol. 19, no. 4, pp. 3079-3088, 2020.

21. Q. Yuan, H. Yu, J. Chen, X. Song and L. Sun, "ADAM10 promotes cell growth, migration, and invasion in osteosarcoma via regulating E-cadherin/beta-catenin signaling pathway and is regulated by miR-122-5p," Cancer Cell Int, vol. 20, pp. 99, 2020.

22. K. Xia, Y. Zhang, S. Cao, Y. Wu, W. Guo, W. Yuan and S. Zhang, "miR-411 regulated ITCH expression and promoted cell proliferation in human hepatocellular carcinoma cells," Biomed Pharmacother, vol. 70, pp. 158-163, 2015.

23. H. Zhang, J. Pu, T. Qi, M. Qi, C. Yang, S. Li, K. Huang, L. Zheng and Q. Tong, "MicroRNA-145 inhibits the growth, invasion, metastasis and angiogenesis of neuroblastoma cells through targeting hypoxia-inducible factor 2 alpha," Oncogene, vol. 33, no. 3, pp. 387-397, 2014.

24. J. Kovalevich and D. Langford, "Considerations for the use of SH-SY5Y neuroblastoma cells in neurobiology," Methods Mol Biol, vol. 1078, pp. 9-21, 2013.

25. G. E. Coggins, A. Farrel, K. S. Rathi, C. M. Hayes, L. Scolaro, J. L. Rokita and J. M. Maris, "YAP1 Mediates Resistance to MEK1/2 Inhibition in Neuroblastomas with Hyperactivated RAS Signaling," Cancer Res, vol. 79, no. 24, pp. 6204-6214, 2019.

26. A. Schramm, J. Koster, Y. Assenov, K. Althoff, M. Peifer, E. Mahlow, A. Odersky, D. Beisser, C. Ernst, A. G. Henssen, H. Stephan, C. Schroder, L. Heukamp, A. Engesser, Y. Kahlert, J. Theissen, B. Hero, F. Roels, J. Altmuller, P. Nurnberg, K. Astrahantseff, C. Gloeckner, K. De Preter, C. Plass, S. Lee, H. N. Lode, K. O. Henrich, M. Gartlgruber, F. Speleman, P. Schmezer, F. Westermann, S. Rahmann, M. Fischer, A. Eggert and J. H. Schulte, "Mutational dynamics between primary and relapse neuroblastomas," Nat Genet, vol. 47, no. 8, pp. 872-877, 2015.

27. A. A. Ahmed, A. D. Mohamed, M. Gener, W. Li and E. Taboada, "YAP and the Hippo pathway in pediatric cancer," Mol Cell Oncol, vol. 4, no. 3, pp. e1295127, 2017.

28. Z. Salah, G. Melino and R. I. Aqeilan, "Negative regulation of the Hippo pathway by E3 ubiquitin ligase ITCH is sufficient to promote tumorigenicity," Cancer Res, vol. 71, no. 5, pp. 2010-2020, 2011.

29. K. C. Ho, Z. Zhou, Y. M. She, A. Chun, T. D. Cyr and X. Yang, "Itch E3 ubiquitin ligase regulates large tumor suppressor 1 stability [corrected]," Proc Natl Acad Sci US A, vol. 108, no. 12, pp. 4870-4875, 2011.

30. Z. L. Luo, H. J. Luo, C. Fang, L. Cheng, Z. Huang, R. Dai, K. Li, F. Z. Tian, T. Wang and L. J. Tang, "Negative correlation of ITCH E3 ubiquitin ligase and miRNA-106b dictates metastatic progression in pancreatic cancer," Oncotarget, vol. 7, no. 2, pp. 1477-1485, 2016.

31. D. Han, Y. Wang, Y. Wang, X. Dai, T. Zhou, J. Chen, B. Tao, J. Zhang and F. Cao, "The Tumor-Suppressive Human Circular RNA CircITCH Sponges miR-330-5p to Ameliorate Doxorubicin-Induced Cardiotoxicity Through Upregulating SIRT6, Survivin, and SERCA2a," Circ Res, vol. 127, no. 4, pp. e108-e125, 2020.

32. F. Li, K. Ma, M. Sun and S. Shi, "Identification of the tumor-suppressive function of circular RNA ITCH in glioma cells through sponging miR-214 and promoting linear ITCH expression," Am J Transl Res, vol. 10, no. 5, pp. 1373-1386, 2018.

33. J. Zhao, K. Zhou, L. Ma and H. Zhang, "MicroRNA-145 overexpression inhibits neuroblastoma tumorigenesis in vitro and in vivo," Bioengineered, vol. 11, no. 1, pp. 219-228, 2020.

34. S. Vijayakumar, G. Liu, H. C. Wen, Y. Abu, R. Chong, H. Nastri, G. G. Bornstein, Z. Q. Pan and S. A. Aaronson, "Extracellular LDLR repeats modulate Wnt signaling activity by promoting LRP6 receptor endocytosis mediated by the Itch E3 ubiquitin ligase," Genes Cancer, vol. 8, no. 7-8, pp. 613-627, 2017.

35. S. Wang, Y. Wu, Y. Xu and X. Tang, "miR-10b promoted melanoma progression through Wnt/beta-catenin pathway by repressing ITCH expression," Gene, vol. 710, pp. 39-47, 2019. 
36. N. Furth and Y. Aylon, "The LATS1 and LATS2 tumor suppressors: beyond the Hippo pathway," Cell Death Differ, vol. 24, no. 9, pp. 1488-1501, 2017.

37. Z. Meng, T. Moroishi and K. L. Guan, "Mechanisms of Hippo pathway regulation," Genes Dev, vol. 30, no. 1, pp. 1-17, 2016.

38. O. Dobrokhotov, M. Samsonov, M. Sokabe and H. Hirata, "Mechanoregulation and pathology of YAP/TAZ via Hippo and non-Hippo mechanisms," Clin Transl Med, vol. 7, no. 1, pp. 23, 2018.

39. X. Shen, X. Xu, C. Xie, H. Liu, D. Yang, J. Zhang, Q. Wu, W. Feng, L. Wang, L. Du, L. Xuan, C. Meng, H. Zhang, W. Wang, Y. Wang, T. Xie and Z. Huang, "YAP promotes the proliferation of neuroblastoma cells through decreasing the nuclear location of p27(Kip1) mediated by Akt," Cell Prolif, vol. 53, no. 2, pp. e12734, 2020.

40. C. Geng, J. Wei and C. Wu, "Yap-Hippo pathway regulates cerebral hypoxia-reoxygenation injury in neuroblastoma N2a cells via inhibiting ROCK1/F-actin/mitochondrial fission pathways," Acta Neurol Belg, vol. 120, no. 4, pp. 879-892, 2020.

41. S. K. Lim, S. Y. Lu, S. A. Kang, H. J. Tan, Z. Li, Z. N. Adrian Wee, J. S. Guan, V. P. Reddy Chichili, J. Sivaraman, T. Putti, A. A. Thike, P. H. Tan, M. Sudol, D. M. Virshup, S. W. Chan, W. Hong and Y. P. Lim, "Wnt Signaling Promotes Breast Cancer by Blocking ITCH-Mediated Degradation of YAP/TAZ Transcriptional Coactivator WBP2," Cancer Res, vol. 76, no. 21, pp. 6278-6289, 2016.

42. Z. Salah, E. Itzhaki and R. I. Aqeilan, "The ubiquitin E3 ligase ITCH enhances breast tumor progression by inhibiting the Hippo tumor suppressor pathway," Oncotarget, vol. 5, no. 21, pp. 10886-10900, 2014.

43. Y. Zhang, W. W. Ta, P. F. Sun, Y. F. Meng and C. Z. Zhao, "Diagnostic and prognostic significance of serum miR-145-5p expression in glioblastoma," Int J Clin Exp Pathol, vol. 12, no. 7, pp. 2536-2543, 2019.

44. Y. Zhang, X. Wen, X. L. Hu, L. Z. Cheng, J. Y. Yu and Z. B. Wei, "Downregulation of miR-145-5p correlates with poor prognosis in gastric cancer," Eur Rev Med Pharmacol Sci, vol. 20, no. 14, pp. 3026-3030, 2016.

45. W. Tang, X. Zhang, W. Tan, J. Gao, L. Pan, X. Ye, L. Chen and W. Zheng, "miR-145-5p Suppresses Breast Cancer Progression by Inhibiting SOX2," J Surg Res, vol. 236, pp. 278-287, 2019.

46. K. W. Kim, J. Qiao, J. Y. Kim, K. Park and D. H. Chung, "Overexpression of microRNA-145 inhibits tumorigenesis through autophagy in chemotherapy and radiation resistant neuroblastoma cells," Oncoscience, vol. 7, no. 1-2, pp. 1-9, 2020 